\title{
Crescimento inicial de ervilha sob diferentes níveis de compactação em dois tipos de solos
}

\section{Initial growth of pea under different compaction levels of two types of soils}

\author{
Aparecida Leonir da Silva ${ }^{2}$, Antônio César Batista Matos ${ }^{2}$, Paulo César Hilst ${ }^{3}$, Helton de Souza Silva ${ }^{4}$
}

Resumo: O manejo do solo pode alterar as propriedades físicas do solo, sendo de grande importância para garantir o estabelecimento de plântulas e produtividade. A inovação de implementos e máquinas agrícolas e excesso de tratos culturais durante o manejo são os principais fatores que interferem nestas características. Assim, objetivou-se avaliar o efeito da compactação de solos com diferentes classes texturais (argilosa e muito argilosa) no crescimento inicial de ervilha. O experimento foi montado em casa de vegetação com sementes de ervilha Itapuã 600 . Cada tratamento contou com 4 repetições de 50 sementes em diferentes graus de compactação dos solos, com aplicações três cargas sobre os solos que resultaram em: argiloso: controle: 0,21, T1: 0,26, T2: 0,28 e T3: 0,33 MPa, textura muito argilosa: controle: 0,40, T1: 0,42, T2: 0,46 e T3: 0,53 MPa. Foram avaliados a porcentagem de emergência, IVE, VE, comprimento de parte aérea e raiz, massa seca de parte aérea, raiz e massa seca total. Para as duas classes texturais a compactação teve influência sobre a massa seca da parte aérea no solo com textura argilosa e para IVE no solo com textura muito argilosa. Porém, ambos não tiveram relação direta com o nível de compactação.

Palavras-chaves: Pisum sativum L.; propriedades físicas do solo; classes texturais.

Abstract: Soil management can alter the physical properties of the soil, being of great importance to ensure adequate levels of crop productivity. The innovation of agricultural implements and machinery and excess cultural practices during handling are the main factors that affect these characteristics. The objective of this study was to evaluate the effect of compaction of soils with different texture classes (clay and heavy clay) on the initial growth of pea. The experiment was conducted in a greenhouse at the Department of Fitotecnia at UFV. Itapuã 600 pea seeds were used. Each treatment consisted of 4 replicates of 50 seeds for each compression level (T1, T2, T3 and without compression) for the two soil textural classes. Were evaluated: emergency, IVE, VE, length of shoot and root dry weight of shoot, root mass and total dry mass. For both textural classes compaction is not observed influence on the parameters analyzed, except for dry weight of shoots in clayey soil, IVE in clayey soil. However, both were not directly related to the level of compression.

Key words: Pisum sativum L., physical properties of soil, textural classes.

\footnotetext{
*Autor para correspondência

Recebido para publicação em 22/05/2015; aprovado em 15/09/2015

${ }^{1}$ Bióloga, Universidade Federal de Viçosa, Viçosa; (31) 3899-2619, aparecidaleonir@gmail.com

${ }^{2}$ Engenheiro Florestal, Universidade Federal de Viçosa, batistamatos@ gmail.com

${ }^{3}$ Engenheiro Agrônomo, Universidade Federal de Viçosa, pchilst@ yahoo.com.br

${ }^{4}$ Engenheiro Agrônomo, Universidade Federal da Paraíba, heltonssilva@gmail.com
} 


\section{INTRODUÇÃO}

O Brasil alcançou nas últimas décadas posição de destaque na agricultura mundial, devido às inovações tecnológicas que permitiram a expansão agrícola (SEVERIANO et al., 2011). Esta evolução tecnológica é direcionada para sistemas padronizados e simplificados de monocultura que demandam práticas de manejo mecanizado, uso de agroquímicos e de irrigação (BALBINO et al., 2011). O preparo do solo, está relacionado com os atributos físicos do solo, tendo influência direta na sua estrutura (HAMZA; ANDERSON, 2005). No entanto, os sistemas de preparo, colheita e manejo causam impactos, como a compactação do solo. Assim, a avaliação das propriedades físicas como a densidade, a macroporosidade (CARNEIRO et al., 2009; LIMA et. al., 2007) e a resistência do solo à penetração (TAVARES FILHO; RIBON, 2008) são relevantes para estimar a capacidade de carga do solo.

A susceptibilidade do solo à compactação apresenta variações, em função das propriedades do solo, como teor de água e textura. A textura influencia o comportamento do solo, quando submetido a pressões externas, pois determina o atrito entre as partículas e o tipo de ligação entre elas. Em geral, quanto maiores as partículas do solo, menor sua compressibilidade e agregação (MACEDO et. al., 2010). Segundo Aguiar (2008), a estrutura do solo pode ser avaliada pela densidade do solo, macro e microporosidade, estabilidade de agregados, resistência à penetração e infiltração da água no solo.

A compactação do solo tem sido um problema no estabelecimento de culturas e da produção em muitas regiões do mundo (SOANE; VAN OUWERKERK, 1994). Apresentando efeitos importantes sobre o crescimento da planta e produção de culturas, podendo ser resultado do uso de máquinas agrícolas no campo. Sob tais condições a densidade do solo aumenta e altera as propriedades (físicas, químicas e biológicas) criando um novo ambiente neste solo.

A compactação do solo é definida como a compressão do solo por forças externas que diminuem o volume de poros, o que causa aumento da densidade do solo (HARRIS, 1971). O aumento da resistência à pressão de compactação do solo, também pode ser entendida como a taxa de microporos do solo, que reduz o crescimento das plantas (MIRANSARI et al., 2007; MIRANSARI et al. 2009).

A ervilha (Pisum sativum L.) é uma hortaliça, pertencente a família Fabaceae, originária do Oriente Médio, sendo um dos alimentos mais produzidos no mundo, devido seu alto valor nutritivo (NASCIMENTO et al., 2007). No Brasil, a cultura adapta-se melhor a regiões de temperatura amena ou nas regiões tropicais com altitudes elevadas (FILGUEIRA, 2008). Porém a participação do Brasil no mercado mundial de ervilhas é inexpressiva. A CEAGESP-SP registrou de janeiro a julho do ano de 2010 a comercialização de 1092 toneladas (AGRIANUAL, 2011).

O uso de alguns tipos de solos e os sistemas de manejo são considerados como causa para aumento na densidade e resistência mecânica dos solos (GLAB; KULIG, 2008; ABID; LAL, 2008; SEVERIANO et al., 2008); tamanho dos poros e diminuição da porosidade total, (SEVERIANO et al., 2008), a redução na absorção de nutrientes e a redução das trocas gasosas (GYSI, 2001) e aumento da capacidade de carga do solo (DIAS JUNIOR et al., 2008).

Diversos estudos demonstram o efeito negativo da compactação dos solos na produção das culturas, diante do exposto, objetivou-se avaliar a emergência de ervilha em solos com diferentes texturas e niveis de compactação.

\section{MATERIAL E MÉTODOS}

O estudo foi realizado no Laboratório de Análise de Sementes do Departamento de Fitotecnia da Universidade Federal de Viçosa.

Foram utilizadas quatro repetições de 50 sementes de Pisum sativum L. (ervilha Itapuã 600), para cada solo, foram coletadas amostras para realização de análise granulométrica. As sementes foram distribuídas em bandejas plásticas (36 x $24 \times 7,5 \mathrm{~cm}$ ), as quais foram preenchidas com dois tipos de solo (textura argilosa ou muito argilosa) e as sementes foram semeadas com $1 \mathrm{~cm}$ de profundidade. Posteriormente, foram aplicadas nos solos diferentes graus de compactação, para obtenção dos três níveis utilizando cargas de pesos conhecidos: 15,30 e $45 \mathrm{Kg}$ por 10 minutos sobre os solos que resultaram em: Argiloso: Controle (sem compactação): 0,21, T1: 0,26, T2: 0,28 e T3: 0,33 MPa, textura muito argilosa: Controle: 0,40, T1: 0,42, T2: 0,46 e T3: 0,53 MPa, definidos em análise, utilizando o método resistência a penetração, com auxilio de um penetrômetro de bancada da marca Marconi, modelo MA 933. Além disso, foram obtidos valores de densidade e porosidade do solo.

Posteriormente as bandejas foram dispostas aleatoriamente em casa de vegetação, com controle de irrigação diária.

Foram enviadas amostras dos solos compactados, para análise física e química (textura do solo, densidade do solo $\left(\mathrm{g} / \mathrm{m}^{3}\right)$, microporosidade $\left(\mathrm{cm}^{3}\right)$, macroporisadade $\left(\mathrm{cm}^{3}\right)$ e porosidade total $\left(\mathrm{cm}^{3}\right)$ ) no Departamento de Solos da Universidade Federal de Viçosa.

Para a avaliação da emergência, foram feitas contagens de emergência de plântulas normais diariamente, até a estabilização de todas as repetições, Velocidade de Emergência (VE) e foi calculado o Índice de Velocidade de Emergência empregando-se a fórmula proposta por Maguire (1962) (Eq. 01).

$$
\operatorname{IVE}=\left(\mathrm{G}_{1} / \mathrm{N}_{1}\right)+\left(\mathrm{G}_{2} / \mathrm{N}_{2}\right)+\ldots+\left(\mathrm{G}_{\mathrm{n}} / \mathrm{N}_{\mathrm{n}}\right)
$$

Onde: IVE = índice de velocidade de emergência;

$\mathrm{G}_{1}, \mathrm{G}_{2}, \mathrm{G}_{\mathrm{n}}=$ número de plântulas normais computadas na primeira contagem, na segunda contagem e na última contagem;

$\mathrm{N}_{1}, \mathrm{~N}_{2}, \mathrm{~N}_{\mathrm{n}}=$ Número de dias da semeadura à primeira contagem, à segunda contagem e à última contagem.

Após a estabilização da emergência, foram efetuadas as medições do comprimento da parte aérea e sistema radicular das plântulas normais com o auxílio de uma régua graduada em $\mathrm{cm}$. Os resultados foram expressos em centímetros por plântula (NAKAGAWA, 1999). Posteriormente foi determinada a massa seca da parte aérea e do sistema radicular, em estufa de ar forçado, a $70{ }^{\circ} \mathrm{C}$, até atingirem peso constante. Os resultados foram expressos em 
miligramas de parte aérea por plântula e em miligramas de sistema radicular por plântula (NAKAGAWA, 1999).

Utilizou-se esquema fatorial (2 classes texturais de solo x 3 níveis de compactação do solo) no delineamento em blocos casualizados com quatro repetições. Os dados foram interpretados estatisticamente, por meio de análise de variância e pelo teste de Tukey a $5 \%$ de probabilidade utilizando o Software Statistica 7.0.

De acordo com a análise granulométrica as amostras de solos foram classificadas de acordo com a textura em argilosa e muito argilosa (Tabela 1) e na Tabela 2 o resultado do teste de resistência à penetração.

Tabela 1 - Resultados da análise granulométrica das amostras de solo.

\begin{tabular}{|c|c|c|c|c|c|}
\hline \multirow{2}{*}{ Solo } & Areia Grossa & Areia Fina & Silte & Argila & \multirow{2}{*}{ Classe Textural } \\
\hline & & $\mathrm{dag} / \mathrm{Kg}$ & & & \\
\hline Solo 1 & 16 & 11 & 18 & 55 & Argilosa \\
\hline Solo 2 & 9 & 8 & 20 & 63 & Muito Argilosa \\
\hline
\end{tabular}

Tabela 2 - Resultados da análise de resistência à penetração das amostras de solo.

\begin{tabular}{cccccc}
\hline Tratamento & Textura solo & Densidade do solo $\left(\mathrm{g} / \mathrm{m}^{3}\right)$ & Microporosidade $\left(\mathrm{cm}^{3}\right)$ & Macroporisadade $\left(\mathrm{cm}^{3}\right)$ & $\begin{array}{c}\text { Porosidade total } \\
\left(\mathrm{cm}^{3}\right)\end{array}$ \\
\hline Controle & Argilosa & 0,80 & 0,42 & 0,28 & 0,70 \\
T1 & Argilosa & 0,83 & 0,40 & 0,30 & 0,70 \\
T2 & Argilosa & 0,80 & 0,39 & 0,31 & 0,70 \\
T3 & Argilosa & 0,84 & 0,42 & 0,28 & 0,70 \\
Controle & Muito argilosa & 1,00 & 0,37 & 0,27 & 0,64 \\
T1 & Muito argilosa & 1,02 & 0,37 & 0,27 & 0,64 \\
T2 & Muito argilosa & 0,96 & 0,37 & 0,29 & 0,66 \\
T3 & Muito argilosa & 1,01 & 0,36 & 0,28 & 0,64 \\
\hline
\end{tabular}

\section{RESULTADOS E DISCUSSÃO}

Dentre todos os parâmetros avaliados para o solo com textura argilosa (Tabela 3 e 4), somente a massa seca da parte aérea teve diferença estatística, no qual o tratamento T3 obteve a maior média, porém não diferiu do controle e do T1. No entanto, T2 não diferiu de T1 e controle (Tabela 4).
Tabela 3 - Valores médios de emergência (E\%), índice de velocidade de emergência (IVE) e velocidade de emergência (VE) de ervilha submetida a diferentes níveis de compactação em solo com textura argilosa.

\begin{tabular}{lccc}
\hline Tratamentos & E \% & IVE & VE \\
\hline T1 & $93,00 \mathrm{a}$ & $6,35 \mathrm{a}$ & $7,50 \mathrm{a}$ \\
T2 & $97,00 \mathrm{a}$ & $7,24 \mathrm{a}$ & $7,46 \mathrm{a}$ \\
T3 & $94,00 \mathrm{a}$ & $6,48 \mathrm{a}$ & $7,40 \mathrm{a}$ \\
Controle & $94,00 \mathrm{a}$ & $6,65 \mathrm{a}$ & $7,16 \mathrm{a}$ \\
\hline CV $(\%)$ & 3,56 & 8,36 & 9,18 \\
\hline
\end{tabular}

* significativo a $5 \%$ de probabilidade pelo teste de F. Médias seguidas de pelos menos uma mesma letra na coluna não diferem entre si pelo teste de Tukey, a $5 \%$ de probabilidade.

Tabela 4 - Valores médios de comprimento da parte aérea (PA), comprimento de raiz (RAIZ), massa seca da parte aérea (MSPA), massa seca de raiz (MSR) e massa seca total (MST) de ervilha submetida a diferentes níveis de compactação em solo com textura argilosa.

\begin{tabular}{llllll}
\hline Tratamentos & PA $(\mathrm{cm})$ & RAIZ $(\mathrm{cm})$ & MSPA $(\mathrm{mg}) *$ & MSR $(\mathrm{mg})$ & MST $(\mathrm{mg})$ \\
\hline T1 & $6,83 \mathrm{a}$ & $20,38 \mathrm{a}$ & $79,15 \mathrm{ab}$ & $73,50 \mathrm{a}$ & $152,65 \mathrm{a}$ \\
T2 & $6,30 \mathrm{a}$ & $20,00 \mathrm{a}$ & $76,32 \mathrm{~b}$ & $74,25 \mathrm{a}$ & $150,58 \mathrm{a}$ \\
T3 & $6,38 \mathrm{a}$ & $20,00 \mathrm{a}$ & $85,44 \mathrm{a}$ & $75,92 \mathrm{a}$ & $161,37 \mathrm{a}$ \\
Controle & $6,00 \mathrm{a}$ & $19,50 \mathrm{a}$ & $81,7 \mathrm{ab}$ & $77,61 \mathrm{a}$ & $159,32 \mathrm{a}$ \\
\hline
\end{tabular}

* significativo a $5 \%$ de probabilidade pelo teste de F. Médias seguidas de pelos menos uma mesma letra na coluna não diferem entre si pelo teste de Tukey, a $5 \%$ de probabilidade.

Segundo Gediga (1991), a matéria seca da parte aérea do milho foi incrementada em $10 \%$ quando aumentou-se a densidade do solo de 1,45 para $1,70 \mathrm{mg} / \mathrm{m}^{3}$ em subsuperfície. Tal fato pode ser explicado pelo aumento da superfície de contato entre raízes e partículas do solo, proporcionando condições adequadas de absorção de água e nutrientes, o que influencia na translocação de nutrientes para a parte aérea.

Rosolem et al. (2002) não verificaram redução da massa da parte aérea do milho submetido a $2 \mathrm{MPa}$ de impedância mecânica do solo, colhido após 25 dias da emergência das plântulas. De acordo com Calonego et al.
(2011), a compactação do solo não reduziu a produção de massa seca da parte aérea e radicular das espécies Dolichos lab lab, Sorghum bicolor L. e Brachiaria ruziziensis. Os autores ainda destacam que na presença de solo compactado, houve aumento no crescimento radicular de Brachiaria ruziziensis na camada intermediária do vaso.

Por outro lado, Silva e Rosolem (2001) observaram que o crescimento da parte aérea de plantas de sorgo granífero foi reduzido em mais de $40 \%$ ao final de 38 dias de cultivo, em solo com uma resistência mecânica da ordem de 1,22 MPa. Entretanto, os resultados destes trabalhos são 
contraditórios, o que reforça os relatos de Bennie (1996) sobre a dificuldade de se estudar o desenvolvimento de plantas em solos compactados.

Dentre todos os parâmetros avaliados para o solo com textura muito argilosa (Tabela 5 e 6), somente o índice de velocidade de emergência (IVE) teve diferença estatística, no qual o controle obteve a maior média, porém não diferiu de T2 e T3. No entanto, T1 não diferiu de T2 e T3 (Tabela 5).
Tabela 5 - Valores médios de emergência (E\%), índice de velocidade de emergência (IVE) e velocidade de emergência (VE) de ervilha submetida a diferentes níveis de compactação em solo com textura muita argilosa.

\begin{tabular}{lccc}
\hline Tratamentos & E \% & IVE $*$ & VE \\
\hline T1 & $82,00 \mathrm{a}$ & $5,07 \mathrm{~b}$ & $8,28 \mathrm{a}$ \\
T2 & $87,00 \mathrm{a}$ & $5,48 \mathrm{ab}$ & $8,19 \mathrm{a}$ \\
$\mathrm{T} 3$ & $80,00 \mathrm{a}$ & $5,1 \mathrm{ab}$ & $8,04 \mathrm{a}$ \\
Controle & $87,00 \mathrm{a}$ & $5,89 \mathrm{a}$ & $7,62 \mathrm{a}$ \\
\hline CV $(\%)$ & 5,42 & 8,69 & 9,71 \\
\hline
\end{tabular}

* significativo a $5 \%$ de probabilidade pelo teste de F. Médias seguidas de pelos menos uma mesma letra na coluna não diferem entre si pelo teste de Tukey, a $5 \%$ de probabilidade.

Tabela 6 - Valores médios de comprimento da parte aérea (PA), comprimento de raiz (RAIZ), massa seca da parte aérea (MSPA), massa seca de raiz (MSR) e massa seca total (MST) de ervilha submetida a diferentes níveis de compactação em solo com textura muita argilosa.

\begin{tabular}{llllll}
\hline Tratamentos & PA $(\mathrm{cm})$ & RAIZ $(\mathrm{cm})$ & MSPA $(\mathrm{mg})$ & MSR $(\mathrm{mg})$ & MST $(\mathrm{mg})$ \\
\hline T1 & $5,00 \mathrm{a}$ & $11,63 \mathrm{a}$ & $46,53 \mathrm{a}$ & $86,91 \mathrm{a}$ & $133,44 \mathrm{a}$ \\
T2 & $5,50 \mathrm{a}$ & $13,25 \mathrm{a}$ & $48,87 \mathrm{a}$ & $96,94 \mathrm{a}$ & $145,81 \mathrm{a}$ \\
T3 & $6,03 \mathrm{a}$ & $12,60 \mathrm{a}$ & $53,09 \mathrm{a}$ & $84,44 \mathrm{a}$ & $137,54 \mathrm{a}$ \\
Controle & $5,58 \mathrm{a}$ & $13,13 \mathrm{a}$ & $52,28 \mathrm{a}$ & $89,90 \mathrm{a}$ & $142,08 \mathrm{a}$ \\
\hline CV $(\%)$ & 9,71 & 10,47 & 10,03 & 9,81 & 7,85 \\
\hline \multicolumn{7}{r}{ * significativo a 5\% de probabilidade pelo teste de F. Médias seguidas de pelos menos uma mesma letra na coluna não diferem entre si pelo teste de }
\end{tabular}
Tukey, a $5 \%$ de probabilidade.

Hyatt et al. (2007); Masaka e Khumbala (2007) observaram que com o aumento da compactação do solo houve diminuição da emergência de soja e café, respectivamente. Segundo Hyatt et al. (2007) uma maior compactação do solo interferiu na viabilidade da sementes, com aumento da mortalidade das sementes. Além disso, sementes germinadas foram incapazes de romper a superfície compactada.

Masaka e Khumbula (2007) observaram que as plantas de café cultivadas em solos compactados tiveram redução da altura do caule, da espessura de caule e da biomassa radicular em comparação ao controle. Os autores concluíram que estes resultados tem relação com a diminuição da porosidade. Resultados similares foram encontrados por Altikat e Celik (2011), que observaram diminuição da emergência de lentilhas com o aumento da resistência do solo. No geral, o nível crítico de compactação do solo varia entre as diferentes culturas.

\section{CONCLUSÕES}

Para o solo com textura argilosa apenas a massa seca da parte aérea diferiu entre os tratamentos. Enquanto para o solo com textura muito argilosa apenas o IVE apresentou diferença entre os tratamentos. Porém, para ambos não foi possível determinar uma relação direta com a compactação. Os demais parâmetros avaliados para as duas classes texturais não sofreram influência da compactação.

\section{REFERÊNCIAS BIBLIOGRÁFICAS}

ABID, M.; LAL, R. Tillage and drainage impact on soil quality. In: Aggregate stability, carbon and nitrogen pools. Soil Tillage Research, Amsterdam, v.100, n. 1-2, p.89-98, 2008.
AGRIANUAL. AGRIANUAL 2011: Anuário da agricultura brasileira. São Paulo: FNP, 2011. 495 p.

AGUIAR, M. I. Qualidade física do solo em sistemas agroflorestais. Viçosa: UFV, 2008, 91 f. Dissertação de mestrado em Solos e nutrição de plantas.

ALTIKAT, S.; CELIK, A. The effects of tillage and intra-row compaction on seedbed properties and red lentil emergence under dry land conditions. Soil and Tillage Research, Amsterdam, v.114, n. 1, p.1-8, 2011.

BALBINO, L.C.; CORDEIRO, L.A.M.; PORFÍRIO-DASILVA, V.; MORAES, A. de; MARTINEZ, G.B.; ALVARENGA, R.C.; KICHEL, A.N.; FONTANELI, R.S.; SANTOS, H.P. dos; FRANCHINI, J.C.; GALERANI, P.R. Evolução tecnológica e arranjos produtivos de sistemas de integração lavoura-pecuáriafloresta no Brasil. Pesquisa Agropecuária Brasileira, Brasília, v.46, n. 10, p.i-xii, 2011.

BENNIE, A. T. P. Growth and mechanical impedance. In: WAISEL, Y.; ESHEL, A.; KAFKAFI, U. Plants Roots: the hidden half. 2. ed. New York: Marcel Dekker, 1996, p. 453-470.

CAlONEGO, J. C.; GOMES, T. C.; SANTOS; C. H.; TIRITAN, C. S. Desenvolvimento de plantas de cobertura em solo compactado. Bioscience Journal, Uberlândia, v. 27, n. 2, p. 289-296, 2011.

CARNEIRO, M. A. C.; SOUZA, E. D.; REIS, E.F.; PEREIRA, H. S.; AZEVEDO, W. R. Atributos físicos, químicos e biológicos de solo de cerrado sob diferentes sistemas de uso e manejo. Revista Brasileira de Ciência do Solo, Campinas, v.33, n.1, p.147-157, 2009. 
DIAS JUNIOR, M.S.; SILVA, S.R.; SANTOS, N.S.; ARAUJO JUNIOR, C.F. Assessment of the soil compaction of two ultisols caused by logging operations. Revista Brasileira de Ciência do Solo, Campinas, v.32, n. 6, p.2245-2253, 2008.

FILGUEIRA, F. A. R. Novo manual de olericultura: agrotecnologia moderna na produção e comercialização de hortaliças. 3. ed. Viçosa: MG, 2008. 421p.

GEDIGA, K. Influence of subsoil compaction on the uptake of $45 \mathrm{Ca}$ from the soil profile and on maize yield. Soil and Tillage Research, Amsterdam, v. 19, n. 2, p. 351-355, 1991.

GLAB, T.; KULIG, B. Effect of mulch and tillage system on soil porosity under wheat (Triticum aestivum). Soil Tillage Research, Amsterdam,v.99, n. 2, p.169-178, 2008.

GYSI, M. Compaction of a Eutric Cambisol under heavy wheel traffic Switzerland: Field data and a critical state soil mechanics model approach. Soil Tillage Research, Amsterdam, v.61, n. 3/4, p.133-142, 2001.

HAMZA, M. A.; ANDERSON, W. K. Soil compaction in cropping systems. A review of the nature, causes and possible solutions. Soil Tillage Research, Amsterdam, v.82, n. 2, p.121-145, 2005.

HARRIS, W.L. The soil compaction process. In: BARNES, K. K. et al. (Ed.) Compaction of agricultural soils. Compaction of agricultural soils. Michigan: ASAE, 1971. 9-44 p.

HYATT, J.; WENDROTH, O.; EGLI, D.B.; TEKRONY, D M. Soil compaction and soybean seedling emergence. Crop Science, Madison, v.47, n. 6, p. 2495-2503, 2007.

LIMA, C. L.R.; REICHERT, J. M.; REINERT, D. J.; SUZUKI, L. E. A. S.; DALBIANCO, L. Densidade crítica ao crescimento de plantas considerando água disponível e resistência à penetração de um ArgissoloVermelho distrófico arênico. Ciência Rural, Santa Maria, v.37, n. 4, p.1166-1169, 2007.

MACEDO, V. R. M.; SILVA, A. J. N.; CABEDA, M. S. V. Influência de tensões compressivas na pressão de précompactação e no índice de compressão do solo. Revista Brasileira de Engenharia Agrícola e Ambiental, Campina Grande, v. 14, n. 8, p.856-862, 2010.

MAGUIRE, J.D. Speed of germination-aid in selection and evaluation for seedling emergence and vigor. Crop Science, Madison, v.2, n.1, p.176-177, 1962.

MASAKA, J.; KLUMBULA, J. The effect of soil compaction levels on germination and biometric characteristics of coffee (Coffee arabica) seedlings in the nursery. International Journal of Agricultural Research, v.2, n. 7, p.581-589, 2007.
MIRANSARI, M.; BAHRAMI, H. A.; REJALI, F.; MALAKOUTI, M. J.; TORABI, H. Using arbuscular mycorrhiza to reduce the stressful effects of soil compaction on corn (Zea mays L.) growth. Soil Biology and Biochemistry, Amsterdam, v.39, n. 8, p.2014-2026, 2007.

MIRANSARI, M.; BAHRAMI, H. A.; REJALI, F.; MALAKOUTI, M. J. Using arbuscular mycorrhiza to reduce the stressful effects of soil compaction on wheat (Triticum aestivum L.) growth. Soil Biology and Biochemistry, Amsterdam, v.40, n.5, p.1197-1206, 2009.

NAKAGAWA, J. Testes de vigor baseados no desempenho das plântulas. In: KRZYZANOWSKI, F. C.; VIEIRA, R. D.; FRANÇA-NETO, J. B. Vigor de sementes: conceitos e testes. Londrina: ABRATES, 1999. cap. 2, p.1-24.

NASCIMENTO, W. M.; FREITAS, R.A.; GOMES, E. m.L.; SOARES, A.S. Metodologia para o teste de envelhecimento acelerado em sementes de ervilha. Horticultura Brasileira, Brasília, v.25, n. 2, p.205-209, 2007.

ROSOLEM, C. A.; FOLONI, J. S. S.; TIRITAN, C. S. Root growth and nutrient accumulation in cover crops as affected by soil compaction. Soil and Tillage Research, Amsterdam, v. 65, n. 1, p. 109-115, 2002.

SEVERIANO, E.C.; OLIVEIRA, G.C.; DIAS JUNIOR, M.S.; OLIVEIRA, L.F.C.; CASTRO, M.B. Pressão de preconsolidação e intervalo hídrico ótimo como indicadores de alterações estruturais de um Latossolo e de um Cambissolo sob cana-de-açúcar. Revista Brasileira de Ciência do Solo, Viçosa, v.32, n.4, p.1419-1427, 2008.

SEVERIANO, E. C.; OLIVEIRA, G. C.; DIAS JUNIOR, M. S.; COSTA, K. A. P.; SILVA, F. G.; FERREIRA FILHO, S. M. Structural changes in latosols of the cerrado region: II - soil compressive behavior and modeling of additional compaction. Revista Brasileira de Ciência do Solo, Viçosa, v.35, n. 3, p.783-791, 2011.

SILVA, R. H.; ROSOLEM, C. A. Crescimento radicular de espécies utilizadas como cobertura decorrente da compactação do solo. Revista Brasileira de Ciência do Solo, Viçosa, v. 25, n. 2, p. 253-260, 2001.

SOANE, B.D.; VAN OUWERKERK, C. Soil compaction problems in world agriculture. In: SOANE, B.D.; VAN OUWERKERK, C. (ed.) Soil compaction in crop production. 1994. p.1-21.

TAVARES FILHO, J.; RIBON, A. A. Resistência do solo a penetração em relação ao número de amostras e ao tipo de amostragem. Revista Brasileira de Ciência do Solo, Viçosa, v.32, n. 2, p. 487-494, 2008. 